\title{
Factors Affecting Drug Retention of Janus kinase Inhibitors in Patients with Rheumatoid Arthritis: The ANSWER Cohort Study
}

Kosuke Ebina ( $\nabla$ k-ebina@ort.med.osaka-u.ac.jp)

Osaka Univeristy, Graduate School of Medicine https://orcid.org/0000-0002-2426-1024

Toru Hirano

Nishinomiya Municipal Hospital

Yuichi Maeda

Osaka University Graduate School of Medicine

Wataru Yamamoto

Kurashiki Sweet Hospital

Motomu Hashimoto

Osaka City University Graduate School of Medicine

Koichi Murata

Graduate School of Medicine, Kyoto University

Akira Onishi

Graduate School of Medicine, Kyoto University

Sadao Jinno

Kobe University Graduate School of Medicine

Ryota Hara

Nara Medical University

Yonsu Son

Kansai Medical University

Hideki Amuro

Kansai Medical University

Tohru Takeuchi

Osaka Medical and Pharmaceutical University

Ayaka Yoshikawa

Osaka Medical and Pharmaceutical University

Masaki Katayama

Osaka Red Cross Hospital

Keiichi Yamamoto

Wakayama Medical University

Yasutaka Okita 
Osaka University Graduate School of Medicine

\section{Makoto Hirao}

Osaka University Graduate School of Medicine

\section{Yuki Etani}

Osaka University Gradutae School of Medicine

\section{Atsushi Kumanogoh}

Osaka University Graduate School of Medicine

\section{Seiji Okada}

Osaka University Graduate School of Medicine

\section{Ken Nakata}

Osaka University Graduate School of Medicine

\section{Research article}

Keywords: ANSWER cohort, Baricitinib, Drug retention, Janus kinase inhibitor, Rheumatoid arthritis, Tofacitinib

Posted Date: August 9th, 2021

DOI: https://doi.org/10.21203/rs.3.rs-744939/v1

License: (c) (i) This work is licensed under a Creative Commons Attribution 4.0 International License. Read Full License

Version of Record: A version of this preprint was published at Scientific Reports on January 7th, 2022. See the published version at https://doi.org/10.1038/s41598-021-04075-0. 


\section{Abstract}

Background: This multi-center, retrospective study aimed to clarify the factors affecting drug retention of the Janus kinase inhibitors (JAKi) baricitinib (BAR) and tofacitinib (TOF) in patients with RA.

Methods: Patients were included as follows: females, $80.6 \%$; age, 60.5 years; DAS28-ESR, 4.3; treated with either BAR $(n=166)$ or TOF $(n=185)$ and concomitant glucocorticoid (prednisolone [PSL] equivalent) 5.3 $\mathrm{mg} /$ day (46.7\%) or methotrexate (MTX) $8.9 \mathrm{mg} /$ week (60.7\%); bDMARDs- or JAKi-switched cases (76.6\%). The reasons for drug discontinuation were classified into four major categories: lack of effectiveness, toxic adverse events, non-toxic reasons and remission. The drug retention rate was estimated at 24 months using the Kaplan-Meier method and adjusted for potential confounders using multivariate Cox proportional hazards modelling.

Results: Adjusted discontinuation rates for the corresponding reasons were as follows: lack of effectiveness (22.3\%), toxic adverse events (13.3\%), non-toxic reasons (7.2\%) and remission ( $0.0 \%)$. Prior use of anti-interleukin-6 receptor antibody (alL-6R) was significantly associated with discontinuation due to lack of effectiveness $(P=0.021)$. Ageing $(P=0.015)$, usage of $P S L \geq 5 \mathrm{mg} /$ day $(P=0.017)$ and female sex $(P=0.041)$ were significantly associated with discontinuation due to toxic adverse events. Factors not associated with treatment discontinuation were: number of prior bDMARDs or JAKi, concomitant MTX usage, different JAKi and prior use of TNF inhibitor, CTLA4-Ig or other JAKi.

Conclusions: Prior use of alL-6R was associated with discontinuation due to lack of effectiveness, while ageing ( $\geq 75$ years), PSL usage $\geq 5 \mathrm{mg} /$ day, and female sex were associated with discontinuation due to toxic adverse events.

\section{Introduction}

The recommendations of the 2019 European League Against Rheumatism (EULAR) stated that the efficacies of anti-interleukin (IL)-6 receptor antibody (alL-6R; tocilizumab and sarilumab), cytotoxic T lymphocyte-associated antigen-4-Ig (CTLA4-Ig; abatacept) and Janus kinase inhibitors (JAKi) such as baricitinib (BAR; a JAK1 and JAK2 inhibitor) and tofacitinib (TOF; a JAK1 and JAK3 inhibitor) are considered equivalent to those of tumour necrosis factor inhibitors (TNFi) in both Phase II and Phase III treatments of rheumatoid arthritis (RA) [1]. The authors reported no significant differences in outcomes between biological disease-modifying antirheumatic drugs (bDMARDs) and JAKi therapy, irrespective of their targets.

JAKi inhibits the JAK-signal transducer and activator of transcription pathways system, which leads to the inhibition of IL-6 and various other cytokines [2]. Five JAKi, including TOF (2013), BAR (2017), peficitinib (2019), upadacitinib (2020) and filgotinib (2020), have been approved for use in Japan-the only country to have approved five JAKi. 
In real-world settings, JAKi tends to be introduced in patients with intolerance to methotrexate (MTX) due to comorbidities or with multiple bDMARDs failures-quite different from those recruited in randomised controlled trials. Therefore, it is of great interest to investigate factors affecting the effectiveness and safety of JAKi in 'difficult-to-treat' RA patients, especially those who were previously treated with TNFi, alL-6R, CTLA4-Ig or another JAKi.

The performance of bDMARDs has increasingly been investigated through recent cohort-based observational studies $[3,4]$ in which drug retention is considered a major index of both treatment safety and effectiveness $[5,6]$. We have recently reported the drug retention rates of bDMARDs [7-12], factors affecting the efficacy of bDMARDs $[13,14]$ and factors affecting the achievement of bDMARDs-free remission [15] on the basis of findings from our cohort. The aim of the present multicenter, retrospective study is to clarify the factors affecting drug retention of a JAKi (BAR or TOF) in real-world settings.

\section{Materials And Methods}

\section{Patients}

The Kansai Consortium for Well-being of Rheumatic Disease Patients (ANSWER) cohort is an observational, multicenter registry of patients with RA in the Kansai district of Japan [7-12]. Data were retrospectively collected from patients who were examined at seven major university-related hospitals (Kyoto University, Osaka University, Osaka Medical College, Kansai Medical University, Kobe University, Nara Medial University and Osaka Red Cross Hospital). RA was diagnosed on the basis of the 1987 RA classification criteria of the American College of Rheumatology (ACR) [16] or the 2010 ACR/EULAR RA classification criteria [17].

Patients who were treated with either BAR or TOF between 2013 and 2020, with complete data on the start and discontinuation dates and the reasons for discontinuation, were included in this study. Additional data were collected, including baseline demographic data (age, sex); disease duration; disease activity (disease activity score in 28 joints using erythrocyte sedimentation rate [DAS28-ESR]); Clinical Disease Activity Index (CDAl) score; concomitant doses (calculated as a blank when not combined) and ratios of MTX and glucocorticoid (GC) (prednisolone [PSL] equivalent); concomitant ratios of other conventional disease-modifying antirheumatic drugs (csDMARDs), such as salazosulfapyridine, bucillamine, iguratimod, tacrolimus and leflunomide; rheumatoid factor (RF) and anti-cyclic citrullinated peptide antibody positivity; and Health Assessment Questionnaire Disability Index score [7-9]. Patients were categorised by age: young, $<65$ years; old, $65-74$ years; and very old, $\geq 75$ years [18] and by concomitant dose of PSL ( $<5$ or $\geq 5 \mathrm{mg} /$ day) [19] because previous reports had demonstrated that these categories are associated with drug retention of bDMARDs and JAKi.

In Japan, public national health insurance covers $70 \%-90 \%$ of medical expense, and bDMARDs or JAKi can be administered at the discretion of attending rheumatologists, in accordance with the Japan College of Rheumatology guidelines [20-22]. The dose of each agent is determined in accordance with the 
manufacturer's recommendation. Drug retention was retrospectively evaluated as the duration until definitive treatment interruption. The reasons for discontinuation were classified into four major categories as follows: 1) lack of effectiveness (including primary and secondary); 2) toxic adverse events (infection, skin or systemic reaction and other toxic events, including haematologic, pulmonary, renal, cardiovascular complications and malignancies); 3) non-toxic reasons (patient preference, change in hospital, desire for pregnancy, etc.); and 4) disease remission [7-9, 11, 12]. Physicians were allowed to cite only one reason for discontinuation.

\section{Statistical analyses}

Differences in baseline clinical characteristics between the groups were assessed using the MannWhitney $U$ test (for continuous variables) and the chi-squared test (for categorical variables). The Kaplan-Meier method was used to examine the survival curves for the agents, as determined by the specified causes. Hazard ratios (HR) for discontinuation of treatment at 24 months were analysed and statistically compared using multivariate Cox proportional hazards modelling [3, 7-9]. The analysis was adjusted for potential confounders that could influence drug retention, as previously described (age; sex; disease duration; concomitant PSL and MTX use; number of switched bDMARDs or JAKi; prior use of TNFi, alL-6R, CTLA4-Ig or other JAKi) [3, 23-26]. Statistical analyses were performed using EZR (Saitama Medical Center, Jichi Medical University, Saitama, Japan), which is a graphical user interface for R software (R Foundation for Statistical Computing, Vienna, Austria) [27]. A P value $<0.05$ was considered statistically significant.

\section{Results}

Table 1 presents the baseline clinical characteristics of the patients at initiation of treatment with each agent (females, 80.6\%; age, 60.5 years; disease duration, 11.0 years; RF positivity, 83.9\%; DAS28-ESR, 4.3; concomitant PSL dose, $5.3 \mathrm{mg} /$ day (ratio, 46.7\%); MTX dose, $8.9 \mathrm{mg} /$ week (ratio, 60.7\%); bDMARDs- or JAKi-switched cases 76.6\%; prior use of TNFi (62.1\%), alL-6R (38.5\%), CTLA4-Ig (28.5\%) and JAKi (13.1\%). Most of the patients who experienced prior JAKi were switched from TOF to BAR $(n=30)$ or from BAR to TOF $(n=8)$. Overall, patients were treated by a low dose and ratio of MTX, and had mostly switched from other bDMARDs or JAKi, suggesting 'difficult-to-treat' backgrounds. 
Table 1

Patients' clinical characteristics at initiation of treatment with each agent

\begin{tabular}{|c|c|c|c|}
\hline Variable & $\begin{array}{l}\text { BAR } \\
(n=166)\end{array}$ & $\begin{array}{l}\text { TOF } \\
(n=185)\end{array}$ & $\begin{array}{l}P \\
\text { value }\end{array}$ \\
\hline Age (years) & $60.2 \pm 13.5$ & $60.7 \pm 13.1$ & 0.87 \\
\hline Female sex (\%) & 86.7 & 75.1 & 0.009 \\
\hline Disease duration (years) & $12.6 \pm 10.6$ & $9.7 \pm 8.3$ & 0.016 \\
\hline RF positivity (\%) & 86.1 & 81.6 & 0.50 \\
\hline ACPA positivity (\%) & 82.0 & 83.1 & 0.93 \\
\hline DAS28-ESR & $4.3 \pm 1.3$ & $4.3 \pm 1.3$ & 0.98 \\
\hline CDAl & $17.2 \pm 11.0$ & $18.8 \pm 11.1$ & 0.16 \\
\hline HAQ-DI & $0.9 \pm 0.7$ & $0.9 \pm 0.8$ & 0.81 \\
\hline PSL use (\%) & 42.8 & 50.3 & 0.19 \\
\hline PSL dose (mg/day) & $4.7 \pm 3.2$ & $5.7 \pm 3.3$ & 0.022 \\
\hline MTX use (\%) & 64.5 & 57.3 & 0.048 \\
\hline MTX dose (mg/week) & $8.7 \pm 3.1$ & $9.2 \pm 3.3$ & 0.35 \\
\hline SASP use (\%) & 11.4 & 23.8 & 0.004 \\
\hline BUC use (\%) & 7.8 & 8.6 & 0.93 \\
\hline IGU use (\%) & 13.3 & 17.8 & 0.30 \\
\hline TAC use (\%) & 15.7 & 9.7 & 0.13 \\
\hline LEF use (\%) & 0.0 & 0.0 & N.A. \\
\hline $\begin{array}{l}\text { bDMARDs or JAKi naive } \\
(\%)\end{array}$ & 22.3 & 24.3 & 0.75 \\
\hline 2nd bDMARDs or JAKi (\%) & 23.5 & 24.3 & 0.86 \\
\hline 3rd bDMARDs or JAKi (\%) & 26.5 & 16.2 & 0.018 \\
\hline $\begin{array}{l}\geq 4 \text { th bDMARDs or JAKi } \\
(\%)\end{array}$ & 27.7 & 35.1 & 0.14 \\
\hline Prior TNFi use (\%) & 57.8 & 65.9 & 0.15 \\
\hline Prior alL-6R use (\%) & 36.1 & 40.5 & 0.46 \\
\hline
\end{tabular}

Values are presented as mean \pm standard deviation or percentage. Differences between the groups were assessed by the Mann-Whitney U test or the chi-squared test. 


\begin{tabular}{|c|c|c|c|}
\hline Variable & $\begin{array}{l}\text { BAR } \\
(n=166)\end{array}$ & $\begin{array}{l}\text { TOF } \\
(n=185)\end{array}$ & $\begin{array}{l}P \\
\text { value }\end{array}$ \\
\hline Prior CTLA4-Ig use (\%) & 31.9 & 25.4 & 0.22 \\
\hline Prior JAKi use (\%) & 20.5 & 6.5 & $<0.001$ \\
\hline Prior JAKi & $\begin{array}{l}\text { TOF }(n=30), \operatorname{BAR}(n=1), \operatorname{PEF}(n= \\
3)\end{array}$ & $\begin{array}{l}\text { TOF }(n=4), \operatorname{BAR}(n= \\
8)\end{array}$ & N.A. \\
\hline
\end{tabular}

In total, the adjusted discontinuation rates for the corresponding reasons at 24 months were as follows: lack of effectiveness (22.3\%), toxic adverse events (13.3\%), non-toxic reasons (7.2\%) and remission $(0.0 \%)$. We further investigated the factors affecting discontinuation due to lack of effectiveness and toxic adverse events, using multivariate Cox proportional hazards modelling. As for discontinuation due to lack of effectiveness, a significant confounder was prior use of alL-6R (HR, 2.07; 95\% confidence interval [CI], 1.12-3.83; $\mathrm{P}=0.021)$; prior use of TNFi tended to increase treatment discontinuation (HR, $1.90 ; 95 \% \mathrm{Cl}, 0.94-3.83 ; \mathrm{P}=0.075$ ) (Table 2). As for discontinuation due to toxic adverse events, significant confounders were ageing $(H R, 1.04 ; 95 \% \mathrm{Cl}, 1.01-1.06 ; P=0.015), P S L$ usage $\geq 5 \mathrm{mg} /$ day $(H R$, $2.21 ; 95 \% \mathrm{Cl}, 1.15-4.23 ; \mathrm{P}=0.017)$ and male sex $(\mathrm{HR}, 0.33 ; 95 \% \mathrm{Cl}, 0.11-0.95 ; \mathrm{P}=0.041)$ (Table 3$)$. 
Table 2

Cox proportional hazard analysis for the risk factors of treatment discontinuation due to lack of effectiveness

\begin{tabular}{|lll|}
\hline Variable & HR $(95 \%$ Cl) & P value \\
\hline Prior alL-6R use (\%) & $2.07(1.12-3.83)$ & 0.021 \\
\hline Prior TNFi use (\%) & $1.90(0.94-3.84)$ & 0.075 \\
\hline Age (years) & $0.99(0.97-1.01)$ & 0.14 \\
\hline Sex & $0.65(0.34-1.21)$ & 0.17 \\
\hline Disease duration (years) & $0.98(0.95-1.01)$ & 0.20 \\
\hline MTX use (\%) & $1.21(0.71-2.04)$ & 0.49 \\
\hline PSL use ( $\geq 5$ mg/day) & $0.86(0.50-1.47)$ & 0.57 \\
\hline Switched number of bDMARDs or JAKi & $0.93(0.71-1.22)$ & 0.58 \\
\hline Difference of JAKi (BAR or TOF) & $1.11(0.64-1.94)$ & 0.70 \\
\hline $\begin{array}{l}\text { Prior JAKi use (\%) } \\
\text { Prior CTLA4-Ig use (\%) }\end{array}$ & $1.13(0.48-2.69)$ & 0.78 \\
\hline $\begin{array}{l}\text { Abbreviations: HR, hazard ratio; Cl, confidence interval; alL-6R, anti-interleukin-6 receptor; TNFi, tumour } \\
\text { necrosis factor inhibitors; MTX, methotrexate; PSL, prednisolone; bDMARDs, biological disease- } \\
\text { modifying antirheumatic drugs; JAKi, Janus kinase inhibitor: BAR, baricitinib; TOF, tofacitinib; CTLA4- } \\
\text { Ig, cytotoxic T lymphocyte-associated antigen-4-Ig. }\end{array}$ & \\
\hline
\end{tabular}


Table 3

Cox proportional hazard analysis for the risk factors of treatment discontinuation due to toxic adverse events

\begin{tabular}{|lll|}
\hline Variable & HR (95\% Cl) & P value \\
\hline Age (years) & $1.04(1.01-1.06)$ & 0.015 \\
\hline PSL use ( $\geq 5 \mathrm{mg}$ /day) & $2.21(1.15-4.23)$ & 0.017 \\
\hline Sex (male) & $0.33(0.11-0.95)$ & 0.041 \\
\hline Disease duration (years) & $1.01(0.98-1.04)$ & 0.64 \\
\hline Prior alL-6R use (\%) & $0.83(0.35-1.97)$ & 0.67 \\
\hline MTX use (\%) & $1.14(0.58-2.27)$ & 0.70 \\
\hline Prior JAKi use (\%) & $1.22(0.41-3.61)$ & 0.72 \\
\hline Difference of JAKi (BAR or TOF) & $0.91(0.45-1.85)$ & 0.79 \\
\hline Prior CTLA4-Ig use (\%) & $0.96(0.42-2.19)$ & 0.93 \\
\hline Switched number of bDMARDs or JAKi & $1.01(0.71-1.46)$ & 0.94 \\
\hline Prior TNFi use (\%) & $1.03(0.41-2.60)$ & 0.96 \\
\hline
\end{tabular}

The adjusted drug retention rates for the corresponding reasons and the statistical differences between the groups were as follows.

Between BAR and TOF, there were no significant differences in the retention rates due to lack of effectiveness (BAR, $84.6 \%$ vs. TOF, $75.9 \% ; \mathrm{P}=0.70$ ) or toxic adverse events (BAR, $82.7 \%$ vs. TOF, $87.5 \%$; $=0.79$; data not shown).

With regard to age, adjusted retention rates due to lack of effectiveness were: young, $75.5 \%$ vs. old, $80.7 \%$ vs. very old, $77.2 \%(P=0.39$; Fig. $1 A)$ and those of toxic adverse events were: young, $89.2 \%$ vs. old, $86.5 \%$ vs. very old, $69.3 \%(P=0.028$; Fig. $1 \mathrm{~B})$. With regard to sex, adjusted retention rates due to lack of effectiveness were: male, $84.9 \%$ vs. female, $75.9 \%(P=0.17$; Fig. $1 \mathrm{C})$ and those of toxic adverse events were: male, $95.4 \%$ vs. female, $83.6 \%(P=0.041$; Fig. 1D). Taken together, although no significant difference was observed in the retention between BAR and TOF, very old patients ( $\geq 75$ years) and female patients showed significantly higher rates of discontinuation due to toxic adverse events, compared with other groups.

On the other hand, regarding concomitant GC dose (PSL equivalent $<5 \mathrm{mg}$ or $\geq 5 \mathrm{mg} /$ day), adjusted retention rates for discontinuation due to lack of effectiveness were PSL $<5 \mathrm{mg} / \mathrm{day}, 76.1 \%$ vs. PSL $\geq 5$ $\mathrm{mg} /$ day, $81.7 \%$ ( $P=0.57$; Fig. $2 \mathrm{~A})$ and those for toxic adverse events were $\mathrm{PSL}<5 \mathrm{mg} / \mathrm{day}, 90.2 \% \mathrm{vs}$. PSL $\geq 5 \mathrm{mg} /$ day, $63.6 \%$ ( $P=0.017$; Fig. 2B). As for the influence of switched number of bDMARDs or JAKi, adjusted retention rates due to lack of effectiveness were: naïve, $68.0 \%$ vs. $2 \mathrm{nd}, 81.5 \%$ vs. $3 \mathrm{rd}, 74.3 \%$ vs. 
4th or more, $81.6 \%$ ( $P=0.61$; Fig. $2 C$ ), and those of toxic adverse events were: naïve, $88.9 \%$ vs. 2 nd, $82.7 \%$ vs. 3 rd, $84.6 \%$ vs. 4 th or more, $90.2 \%(P=0.94$; Fig. $2 D)$. Although the number of switched bDMARDs or JAKi did not affect subsequent JAKi retention, concomitant PSL $\geq 5 \mathrm{mg} /$ day was significantly associated with higher treatment discontinuation due to toxic adverse events.

Regarding prior TNFi treatment, the adjusted retention rates due to lack of effectiveness were: without prior TNFi, $82.6 \%$ vs. with prior TNFi, 73.5\% ( $P=0.075$; Fig. $3 \mathrm{~A})$ and those of toxic adverse events were: without prior TNFi, $85.9 \%$ vs. with prior TNFi, 87.1\% ( $P=0.96$; data not shown). As for prior alL-6R treatment, the adjusted retention rates due to lack of effectiveness were: without prior alL-6R, $82.3 \% \mathrm{vs}$. with prior alL-6R, 68.1\% $(P=0.021$; Fig. 3B) and those of toxic adverse events were: without prior alL-6R, $85.1 \%$ vs. with prior alL-6R, $89.0 \%(P=0.67$; data not shown). Regarding prior CTLA4-Ig treatment, the adjusted retention rates due to lack of effectiveness were: without prior CTLA4-Ig, 76.3\% vs. with prior CTLA4-Ig, 81.0\% ( $P=0.97$; Fig. $3 \mathrm{C}$ ) and those of toxic adverse events were: without prior CTLA4-Ig, 87.9\% vs. with prior CTLA4-Ig, 84.3\%; P = 0.93; data not shown). As for prior JAKi treatment, the adjusted retention rates due to lack of effectiveness were: without prior JAKi, $77.4 \%$ vs. with prior JAKi, $81.8 \%$ (P = 0.78 ; Fig. 3D) and those of toxic adverse events were: without prior JAKi, $87.2 \%$ vs. with prior JAKi, $80.8 \%$ $(P=0.72$; data not shown). Taken together, prior treatment with alL-6R showed significantly lower retention rate due to lack of effectiveness than without prior alL-6R treatment. Prior treatment with TNFi, CTLA4-Ig or other JAKi did not significantly affect JAKi treatment retention.

\section{Discussion}

Regarding the difference between BAR and TOF, a previous meta-analysis revealed that, in patients with inadequate response to csDMARDs or bDMARDs, BAR and TOF were similarly efficacious [28], which accords with the results of the present study.

Concerning the effect of ageing, the RA-BUILD and RA-BEAM studies of BAR showed similar clinical efficacy between young ( $<50$ years) and old ( $\geq 65$ years) patients [29], similar to the phase III and longterm extension studies of TOF [30]. However, elderly patients ( $\geq 65$ years) tended to show a higher rate of discontinuation of BAR treatment due to adverse events ( $8.8 \%$ ) than younger patients ( $<50$ years, $2.3 \%$ ) [29], similar to results for TOF [30]. Greater age was associated with increased risk of herpes zoster (HZ) [31], major adverse cardiovascular events [32] and gastrointestinal perforation in TOF treatment [33]. Thus, in JAKi treatment, ageing may not affect efficacy but may attenuate safety.

With regard to sex, females had higher risk of $\mathrm{HZ}$ compared with males in TOF treatment [30]. $\mathrm{HZ}$ is one of the most frequent adverse events in JAKi treatment, and has higher incidence in Japan, compared with western countries [34]. This may lead to the higher rate of discontinuation due to toxic adverse events in our present study. RA disease activity tend to be higher in female, whereas clinical response to csDMARDs and bDMARDs appears to be better in male [35]. In our present study, although the baseline disease activities (DAS28-ESR and CDAI) were similar (data not shown), drug retention of JAKi due to 
lack of effectiveness tended to be higher in male compared to female. This tendency may be similar among these anti-rheumatic agents.

Concomitant use of GC with TOF did not affect clinical or radiographic efficacy [36]. However, the occurrence of $\mathrm{HZ}$ doubled in oral GC use [30]; oral GC (> $7.5 \mathrm{mg} /$ day of PSL) was a risk factor for serious infections (including $\mathrm{HZ}$ ) in TOF treatment [31]. In the present study, patients with PSL $\geq 5 \mathrm{mg} /$ day were at higher risk of toxic adverse events, similar to the results for bDMARDs in Japanese RA patients $[19,37]$.

Another concern is whether the number or mode of action of prior bDMARDs or JAKi may affect the drug retention of JAKi. Although improvement in disease activity was greatest in the bDMARDs-naïve group, both BAR and TOF were effective in patients refractory to multiple bDMARDs [38]. In addition, prior use of bDMARDs did not affect the clinical efficacy of BAR [39]; the clinical efficacy of BAR was similar regardless of previous multiple bDMARD use [40]. Concerning the mode of action of prior bDMARDs or JAKi, prior use of non-TNFi ( $n=31$, including alL-6R and CTLA4-Ig) or JAKi was associated with diminished improvement of DAS28-C-reactive protein (CRP) in BAR treatment [40]. However, non-TNFi (such as alL-6R and JAKi) may overly downregulate CRP levels by inhibiting IL- 6 signalling regardless of its actual disease activity. Therefore, using DAS28-CRP to evaluate disease activity in alL-6R or JAKi treatment may overestimate their clinical response, and also underestimate following treatment response. Considering the underlying mechanisms, BAR inhibits JAK1 and JAK2 signalling, while TOF inhibits JAK1 and JAK3 signalling, which are mainly involved in IL-6 production [2]. Thus, patients who showed inadequate response to alL-6R may not be fully rescued by BAR or TOF. However, JAK2 is also involved in Granulocyte Macrophage Colony-Stimulating Factor (GM-CSF), which initiates arthritis and pain [41], and interferon-y production, which activates macrophages. JAK3 is also involved in IL-2 and IL-21 production, which promote T-cell activation and differentiation, and play important roles in the pathology of RA [42]. Thus, some patients who are dominated by cytokines other than IL-6 may be rescued by switching between BAR and TOF, although further detailed examinations are required.

MTX inhibits not only IL-6 but also inhibits IL-1, matrix metalloproteinases and RF, which play important roles in joint destruction [43]. Indeed, BAR monotherapy was inferior to BAR + MTX in radiographic progression [44]. However, drug retention of BAR due to ineffectiveness [45] and also drug retention of BAR and TOF in our present study were not significantly affected by concomitant MTX. Taken together, the effectiveness of JAKi in inhibiting joint destruction may be superior in combination with MTX, although drug retention based on clinical settings may be similar compared with monotherapy. The effectiveness of low-dose MTX in Japanese populations should be considered. Intra-erythrocyte MTXpolyglutamate concentration, which is considered a useful biomarker of MTX efficacy, was $65 \mathrm{nmol} / \mathrm{L}$ with MTX of $13.4 \mathrm{mg} /$ week in patients from the United States, and reached $94 \mathrm{nmol} / \mathrm{L}$ with MTX of 10.3 $\mathrm{mg} /$ week in Japanese patients [46].

The limitations of the present study are as follows. First, although patients were followed by experienced senior rheumatologist of university-related hospitals, the reasons for discontinuation depended on the decisions of different physicians without standardised criteria. Second, according to the Japanese 
guidelines, TNFi, alL-6R, or CTLA4-Ig are equally recommended in patients who showed inadequate response to csDMARDs, and JAKi (TOF) can be recommended in patients who showed inadequate response to bDMARDs, which may differ from that of western countries and also affected the results. Third, as the initial dose of each agent was determined according to the manufacturer's recommendations, minor changes of dose of each agent during the period couldn't be monitored. Fourth, comorbidities, which can potentially affect drug retention, were not evaluated. Fifth, a relatively small number of prior JAKi-experienced patients may have affected the results. Sixths, the U.S. Food and Drug Administration recently alerted that increased risk of serious cardiovascular events and malignancy of TOF compared to TNFi, which may affect the results of long-term treatment. However, the strength of this study is that it is the first to evaluate factors affecting plural JAKi retention, by adjusting clinical backgrounds according to prior history of TNFi, alL-6R, CTLA4-Ig and JAKi, especially in "difficult-to-treat" RA patients who may not be included in randomised controlled trials.

\section{Conclusions}

In BAR or TOF treatment, a significant negative confounder of discontinuation due to lack of effectiveness was prior use of alL-6R. As for discontinuation due to toxic adverse events, significant negative confounders were ageing ( $\geq 75$ years), concomitant $P S L \geq 5 \mathrm{mg} /$ day and female sex. These novel findings may provide new insight for the management of JAKi in clinical practice.

\section{Abbreviations}

N.A., not applicable; BAR, baricitinib; TOF, tofacitinib; RF, rheumatoid factor; ACPA, anticyclic citrullinated peptide antibody; DAS28-ESR, Disease Activity Score in 28 joints using erythrocyte sedimentation rate; CDAl, clinical disease activity index; HAQ-DI, Health Assessment Questionnaire disability index; PSL, prednisolone; MTX, methotrexate; SASP, salazosulfapyridine; BUC, bucillamine; IGU, iguratimod; TAC, tacrolimus; LEF, leflunomide; bDMARDs, biological disease-modifying antirheumatic drugs; JAKi, Janus kinase inhibitor

TNFi, tumour necrosis factor inhibitors; alL-6R, anti-interleukin-6 receptor; CTLA4-Ig, cytotoxic T lymphocyte-associated antigen-4-Ig; PEF, peficitinib.

\section{Declarations}

\section{Acknowledgments}

We thank all the medical staff at all the institutions who participated in the ANSWER cohort for providing the data.

\section{Availability of data and materials}


The datasets used and/or analyzed in the current study are available from the corresponding author on reasonable request.

\section{Authors' contributions}

KE was responsible for conception and design. KE, TH, YM, YO, MH, KM, AO, SJ, RH, TT, AY, YS, HA, MK, and $E Y$ contributed to data extraction and interpretation. $K E, W Y$, and $K Y$ contributed to the design and conduction of statistical analysis. $\mathrm{KE}$ and $\mathrm{MH}$ prepared the manuscript. $\mathrm{AK}, \mathrm{MH}, \mathrm{SO}$, and $\mathrm{KN}$ supervised the manuscript. All the authors read and approved the final manuscript.

\section{Ethical approval}

The representative facility of this registry was Kyoto University, and this observational study was conducted in accordance with the Declaration of Helsinki, with the approval of the ethics committees of the following seven institutes: Kyoto University (2016-03-24/approval No. R053), Osaka University (201511-04/approval No. 15300), Osaka Medical College (2014-07-14/approval No. 1529), Kansai Medical University (2017-11-21/approval No. 2014625), Kobe University (2015-03-20/approval No. 1738), Nara Medial University (2018-01-23/approval No. 1692), and Osaka Red Cross Hospital (2015-09-01/approval No. 644). The board of the Osaka University Hospital Ethics Committee waived the requirement for patient informed consent because of the anonymous nature of the data. Written informed consent was obtained from the participants in other institutes.

\section{Consent for publication}

Not applicable.

\section{Conflict of interest}

KE is affiliated with the Department of Musculoskeletal Regenerative Medicine, Osaka University, Graduate School of Medicine, which is supported by Taisho. KE has received research grants from Abbie, Amgen, Asahi-Kasei, Astellas, Chugai, Eisai, Mitsubishi-Tanabe, Ono Pharmaceutical, Teijin Pharma, and UCB Japan. KE has received payments for lectures from Abbie, Amgen, Asahi-Kasei, Astellas, Ayumi, Bristol-Myers Squibb, Chugai, Eisai, Eli Lilly, Janssen, Mitsubishi-Tanabe, Ono Pharmaceutical, Pfizer, Sanofi, and UCB Japan. TH received a research grant and/or speaker fee from Astellas, Chugai, GlaxoSmithKline, Nippon Shinyaku, and Eisai. YM received a research grant and/or speaker fee from Eli Lilly, Chugai, Pfizer, Bristol-Myers Squibb, and Mitsubishi-Tanabe. MHashimoto received a research grant and/or speaker fee from Mitsubishi-Tanabe, Eisai, Eli Lilly, Bristol-Myers Squibb, and Novartis Pharma. $\mathrm{KM}$ is affiliated with a department that is financially supported by four pharmaceutical companies (AsahiKasei, Mitsubishi-Tanabe, Chugai, Ayumi, and UCB Japan) and the city government (Nagahama City). KM 
received a speaker fee from Eisai. TT is affiliated with a department that is financially supported by six pharmaceutical companies (Mitsubishi-Tanabe, Chugai, Ayumi, Astellas, Eisai, and Takeda). TT received a research grant from Chugai, cover letter and a speaker fee from Astellas, Chugai, Eisai, MitsubishiTanabe, AbbVie, Bristol-Myers Squibb, Ayumi, Daiichi Sankyo, Eisai, Takeda, and Asahi-Kasei. AO received a speaker fee from Chugai, Ono Pharmaceutical, Eli Lilly, Mitsubishi-Tanabe, Asahi-Kasei, and Takeda. SJ has received speaking fees from AbbVie, Asahi-Kasei, Bristol-Myers Squib, Chugai, Eisai, Eli Lilly, Janssen Pharmaceutical, Mitsubishi-Tanabe, and Ono Pharmaceutical. RH received a speaker fee from AbbVie. MHirao received a speaker fee from Astellas, Ono Pharmaceutical, Eli Lilly, Mitsubishi-Tanabe, Pfizer, Ayumi, and Takeda. AK received a research grant and/or speaker fee from Mitsubishi-Tanabe, Chugai, Eisai, Asahi-Kasei, Astellas, AbbVie, Bristol-Myers Squibb, Ono Pharmaceutical, and Pfizer. KN has received a research grant from Astellas, and supervises the Department of Musculoskeletal Regenerative Medicine, Osaka University, Graduate School of Medicine, which is supported by Taisho. WY, YS, HA, AY, $\mathrm{MK}, \mathrm{KY}, \mathrm{YO}, \mathrm{EY}$, and $\mathrm{SO}$ have no financial conflicts of interest to disclose concerning this manuscript. These companies had no role in the study design, data collection, data analysis, data interpretation, and preparation of the manuscript.

\section{Funding}

The study reported in this publication uses the ANSWER Cohort, was supported by grants from 11 pharmaceutical companies (AbbVie GK, Asahi-Kasei, Ayumi, Chugai, Eisai, Eli Lilly, Janssen K.K, Ono, Sanofi K.K, Teijin Healthcare, and UCB Japan) and an information technology service company (CAC). This study was conducted as an investigator-initiated study. These companies had no roles in the study design, data collection, data analysis, data interpretation, or writing of the report.

\section{References}

1. Smolen JS, Landewe RBM, Bijlsma JWJ, Burmester GR, Dougados M, Kerschbaumer A, Mclnnes IB, Sepriano A, van Vollenhoven RF, de Wit M, et al. EULAR recommendations for the management of rheumatoid arthritis with synthetic and biological disease-modifying antirheumatic drugs: 2019 update. Ann Rheum Dis. 2020;79(6):685-99.

2. Winthrop KL. The emerging safety profile of JAK inhibitors in rheumatic disease. Nat Rev Rheumatol. 2017;13(4):234-43.

3. Du Pan SM, Dehler S, Ciurea A, Ziswiler HR, Gabay C, Finckh A. Comparison of drug retention rates and causes of drug discontinuation between anti-tumor necrosis factor agents in rheumatoid arthritis. Arthritis Rheum. 2009;61(5):560-8.

4. Favalli EG, Pregnolato F, Biggioggero M, Becciolini A, Penatti AE, Marchesoni A, Meroni PL. TwelveYear Retention Rate of First-Line Tumor Necrosis Factor Inhibitors in Rheumatoid Arthritis: Real-Life Data From a Local Registry. Arthritis Care Res (Hoboken). 2016;68(4):432-9. 
5. Hyrich KL, Watson KD, Lunt M, Symmons DP. Changes in disease characteristics and response rates among patients in the United Kingdom starting anti-tumour necrosis factor therapy for rheumatoid arthritis between 2001 and 2008. Rheumatology. 2011;50(1):117-23.

6. Neovius M, Arkema EV, Olsson H, Eriksson JK, Kristensen LE, Simard JF, Askling J. Drug survival on TNF inhibitors in patients with rheumatoid arthritis comparison of adalimumab, etanercept and infliximab. Ann Rheum Dis. 2015;74(2):354-60.

7. Ebina K, Hashimoto M, Yamamoto W, Hirano T, Hara R, Katayama M, Onishi A, Nagai K, Son Y, Amuro $\mathrm{H}$, et al. Drug tolerability and reasons for discontinuation of seven biologics in elderly patients with rheumatoid arthritis -The ANSWER cohort study. PLoS One. 2019;14(5):e0216624.

8. Ebina K, Hashimoto M, Yamamoto W, Hirano T, Hara R, Katayama M, Onishi A, Nagai K, Son Y, Amuro $\mathrm{H}$, et al. Drug tolerability and reasons for discontinuation of seven biologics in 4466 treatment courses of rheumatoid arthritis-the ANSWER cohort study. Arthritis Res Ther. 2019;21(1):91.

9. Ebina K, Hashimoto M, Yamamoto W, Ohnishi A, Kabata D, Hirano T, Hara R, Katayama M, Yoshida S, Nagai $K$, et al. Drug retention and discontinuation reasons between seven biologics in patients with rheumatoid arthritis -The ANSWER cohort study. PLoS One. 2018;13(3):e0194130.

10. Ebina K, Hirano T, Maeda Y, Yamamoto W, Hashimoto M, Murata K, Onishi A, Jinno S, Hara R, Son Y, et al: Drug retention of sarilumab, baricitinib, and tofacitinib in patients with rheumatoid arthritis: the ANSWER cohort study. Clin Rheumatol 2021.

11. Ebina K, Hirano T, Maeda Y, Yamamoto W, Hashimoto M, Murata K, Takeuchi T, Nagai K, Son Y, Amuro $\mathrm{H}$, et al. Drug retention of secondary biologics or JAK inhibitors after tocilizumab or abatacept failure as first biologics in patients with rheumatoid arthritis -the ANSWER cohort study. Clin Rheumatol. 2020;39(9):2563-72.

12. Ebina K, Hirano T, Maeda Y, Yamamoto W, Hashimoto M, Murata K, Takeuchi T, Shiba H, Son Y, Amuro $\mathrm{H}$, et al. Drug retention of 7 biologics and tofacitinib in biologics-naive and biologics-switched patients with rheumatoid arthritis: the ANSWER cohort study. Arthritis Res Ther. 2020;22(1):142.

13. Jinno S, Onishi A, Dubreuil M, Hashimoto M, Yamamoto W, Murata K, Takeuchi T, Kotani T, Maeda Y, Ebina $\mathrm{K}$, et al. Comparison of the drug retention and reasons for discontinuation of tumor necrosis factor inhibitors and interleukin-6 inhibitors in Japanese patients with elderly-onset rheumatoid arthritis-the ANSWER cohort study. Arthritis Res Ther. 2021;23(1):116.

14. Maeda Y, Hirano T, Ebina K, Hara R, Hashimoto M, Yamamoto W, Murakami K, Kotani T, Hata K, Son $Y$, et al. Comparison of efficacy between anti-IL- 6 receptor antibody and other biological diseasemodifying antirheumatic drugs in the patients with rheumatoid arthritis who have knee joint involvement: the ANSWER cohort, retrospective study. Rheumatol Int. 2021;41(7):1233-41.

15. Hashimoto M, Furu M, Yamamoto W, Fujimura T, Hara R, Katayama M, Ohnishi A, Akashi K, Yoshida $\mathrm{S}$, Nagai $\mathrm{K}$, et al. Factors associated with the achievement of biological disease-modifying antirheumatic drug-free remission in rheumatoid arthritis: the ANSWER cohort study. Arthritis Res Ther. 2018;20(1):165. 
16. Arnett FC, Edworthy SM, Bloch DA, McShane DJ, Fries JF, Cooper NS, Healey LA, Kaplan SR, Liang $\mathrm{MH}$, Luthra HS, et al. The American Rheumatism Association 1987 revised criteria for the classification of rheumatoid arthritis. Arthritis Rheum. 1988;31(3):315-24.

17. Aletaha D, Neogi T, Silman AJ, Funovits J, Felson DT, Bingham CO 3rd, Birnbaum NS, Burmester GR, Bykerk VP, Cohen MD, et al. 2010 rheumatoid arthritis classification criteria: an American College of Rheumatology/European League Against Rheumatism collaborative initiative. Ann Rheum Dis. 2010;69(9):1580-8.

18. Lahaye C, Soubrier M, Mulliez A, Bardin T, Cantagrel A, Combe B, Dougados M, Flipo RM, Le Loet X, Shaeverbeke $\mathrm{T}$, et al. Effectiveness and safety of abatacept in elderly patients with rheumatoid arthritis enrolled in the French Society of Rheumatology's ORA registry. Rheumatology. 2016;55(5):874-82.

19. Koike T, Harigai M, Inokuma S, Ishiguro N, Ryu J, Takeuchi T, Takei S, Tanaka Y, Sano Y, Yaguramaki $\mathrm{H}$, et al. Effectiveness and safety of tocilizumab: postmarketing surveillance of 7901 patients with rheumatoid arthritis in Japan. J Rheumatol. 2014;41(1):15-23.

20. Kawahito Y. [Guidelines for the management of rheumatoid arthritis]. Nihon Rinsho. 2016;74(6):93943.

21. Koike R, Harigai M, Atsumi T, Amano K, Kawai S, Saito K, Saito T, Yamamura M, Matsubara T, Miyasaka N. Japan College of Rheumatology 2009 guidelines for the use of tocilizumab, a humanized anti-interleukin-6 receptor monoclonal antibody, in rheumatoid arthritis. Mod Rheumatol. 2009;19(4):351-7.

22. Koike R, Takeuchi T, Eguchi K, Miyasaka N. Update on the Japanese guidelines for the use of infliximab and etanercept in rheumatoid arthritis. Mod Rheumatol. 2007;17(6):451-8.

23. Favalli EG, Biggioggero M, Marchesoni A, Meroni PL. Survival on treatment with second-line biologic therapy: a cohort study comparing cycling and swap strategies. Rheumatology. 2014;53(9):1664-8.

24. Gabay C, Riek M, Scherer A, Finckh A. Effectiveness of biologic DMARDs in monotherapy versus in combination with synthetic DMARDs in rheumatoid arthritis: data from the Swiss Clinical Quality Management Registry. Rheumatology. 2015;54(9):1664-72.

25. Greenberg JD, Reed G, Decktor D, Harrold L, Furst D, Gibofsky A, Dehoratius R, Kishimoto M, Kremer JM. A comparative effectiveness study of adalimumab, etanercept and infliximab in biologically naive and switched rheumatoid arthritis patients: results from the US CORRONA registry. Ann Rheum Dis. $2012 ; 71(7): 1134-42$.

26. Jorgensen TS, Kristensen LE, Christensen R, Bliddal H, Lorenzen T, Hansen MS, Ostergaard M, Jensen $J$, Zanjani L, Laursen T, et al. Effectiveness and drug adherence of biologic monotherapy in routine care of patients with rheumatoid arthritis: a cohort study of patients registered in the Danish biologics registry. Rheumatology. 2015;54(12):2156-65.

27. Kanda Y. Investigation of the freely available easy-to-use software 'EZR' for medical statistics. Bone Marrow Transplant. 2013;48(3):452-8. 
28. Bae SC, Lee YH. Comparison of the efficacy and safety of tofacitinib and baricitinib in patients with active rheumatoid arthritis: a Bayesian network meta-analysis of randomized controlled trials. $Z$ Rheumatol. 2019;78(6):559-67.

29. Fleischmann R, Alam J, Arora V, Bradley J, Schlichting DE, Muram D, Smolen JS. Safety and efficacy of baricitinib in elderly patients with rheumatoid arthritis. RMD Open. 2017;3(2):e000546.

30. Curtis JR, Xie F, Yang S, Bernatsky S, Chen L, Yun H, Winthrop K. Risk for Herpes Zoster in TofacitinibTreated Rheumatoid Arthritis Patients With and Without Concomitant Methotrexate and Glucocorticoids. Arthritis Care Res (Hoboken). 2019;71(9):1249-54.

31. Cohen S, Radominski SC, Gomez-Reino JJ, Wang L, Krishnaswami S, Wood SP, Soma K, Nduaka Cl, Kwok K, Valdez $\mathrm{H}$, et al. Analysis of infections and all-cause mortality in phase II, phase III, and longterm extension studies of tofacitinib in patients with rheumatoid arthritis. Arthritis Rheumatol. 2014;66(11):2924-37.

32. Charles-Schoeman C, DeMasi R, Valdez H, Soma K, Hwang LJ, Boy MG, Biswas P, Mclnnes IB. Risk Factors for Major Adverse Cardiovascular Events in Phase III and Long-Term Extension Studies of Tofacitinib in Patients With Rheumatoid Arthritis. Arthritis Rheumatol. 2019;71(9):1450-9.

33. Xie F, Yun H, Bernatsky S, Curtis JR. Brief Report: Risk of Gastrointestinal Perforation Among Rheumatoid Arthritis Patients Receiving Tofacitinib, Tocilizumab, or Other Biologic Treatments. Arthritis Rheumatol. 2016;68(11):2612-7.

34. Harigai M, Takeuchi T, Smolen JS, Winthrop KL, Nishikawa A, Rooney TP, Saifan CG, Issa M, Isaka Y, Akashi N, et al. Safety profile of baricitinib in Japanese patients with active rheumatoid arthritis with over 1.6 years median time in treatment: An integrated analysis of Phases 2 and 3 trials. Mod Rheumatol. 2020;30(1):36-43.

35. Favalli EG, Biggioggero M, Crotti C, Becciolini A, Raimondo MG, Meroni PL. Sex and Management of Rheumatoid Arthritis. Clin Rev Allergy Immunol. 2019;56(3):333-45.

36. Charles-Schoeman C, van der Heijde D, Burmester GR, Nash P, Zerbini CAF, Connell CA, Fan H, Kwok K, Bananis E, Fleischmann R. Effect of Glucocorticoids on the Clinical and Radiographic Efficacy of Tofacitinib in Patients with Rheumatoid Arthritis: A Posthoc Analysis of Data from 6 Phase III Studies. J Rheumatol. 2018;45(2):177-87.

37. Mori S, Yoshitama T, Hidaka T, Sakai F, Hasegawa M, Hashiba Y, Suematsu E, Tatsukawa H, Mizokami A, Yoshizawa S, et al. Comparative risk of hospitalized infection between biological agents in rheumatoid arthritis patients: A multicenter retrospective cohort study in Japan. PLoS One. 2017;12(6):e0179179.

38. Fitton J, Melville AR, Emery P, Nam JL, Buch MH. Real-world single centre use of JAK inhibitors across the rheumatoid arthritis pathway. Rheumatology (Oxford) 2020.

39. Spinelli FR, Ceccarelli F, Garufi C, Duca I, Mancuso S, Cipriano E, Dell'Unto E, Alessandri C, Di Franco $M$, Perricone $C$, et al: Effectiveness and safety of baricitinib in rheumatoid arthritis: a monocentric, longitudinal, real-life experience. Clin Exp Rheumatol 2020. 
40. Takahashi N, Asai S, Kobayakawa T, Kaneko A, Watanabe T, Kato T, Nishiume T, Ishikawa H, Yoshioka Y, Kanayama Y, et al. Predictors for clinical effectiveness of baricitinib in rheumatoid arthritis patients in routine clinical practice: data from a Japanese multicenter registry. Sci Rep. 2020;10(1):21907.

41. Hirota K, Hashimoto M, Ito Y, Matsuura M, Ito H, Tanaka M, Watanabe H, Kondoh G, Tanaka A, Yasuda K, et al. Autoimmune Th17 Cells Induced Synovial Stromal and Innate Lymphoid Cell Secretion of the Cytokine GM-CSF to Initiate and Augment Autoimmune Arthritis. Immunity. 2018;48(6):1220-32 e1225.

42. Aletaha D, Smolen JS. Diagnosis and Management of Rheumatoid Arthritis: A Review. JAMA. 2018;320(13):1360-72.

43. Swierkot J, Szechinski J. Methotrexate in rheumatoid arthritis. Pharmacol Rep. 2006;58(4):473-92.

44. Fleischmann R, Schiff M, van der Heijde D, Ramos-Remus C, Spindler A, Stanislav M, Zerbini CA, Gurbuz S, Dickson C, de Bono S, et al. Baricitinib, Methotrexate, or Combination in Patients With Rheumatoid Arthritis and No or Limited Prior Disease-Modifying Antirheumatic Drug Treatment. Arthritis Rheumatol. 2017;69(3):506-17.

45. Guidelli GM, Viapiana O, Luciano N, De Santis M, Boffini N, Quartuccio L, Birra D, Conticini E, Chimenti MS, Bazzani C, et al: Efficacy and safety of baricitinib in 446 patients with rheumatoid arthritis: a real-life multicentre study. Clin Exp Rheumatol 2020.

46. Takahashi C, Kaneko Y, Okano Y, Taguchi H, Oshima H, Izumi K, Yamaoka K, Takeuchi T. Association of erythrocyte methotrexate-polyglutamate levels with the efficacy and hepatotoxicity of methotrexate in patients with rheumatoid arthritis: a 76-week prospective study. RMD Open. 2017;3(1):e000363.

\section{Figures}


a

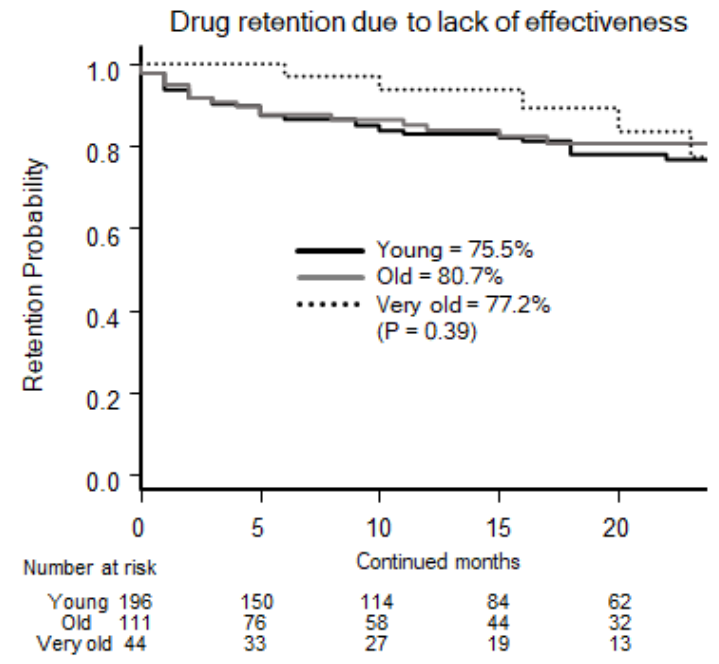

C

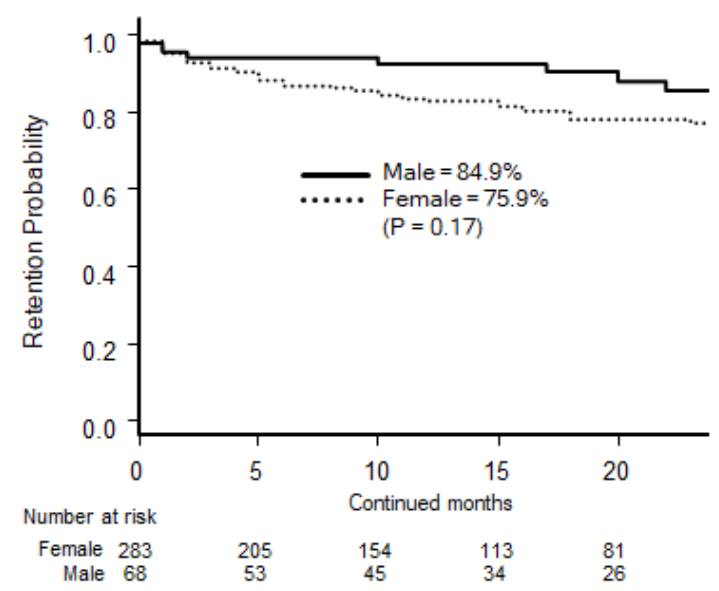

b

Drug retention due to toxic adverse events

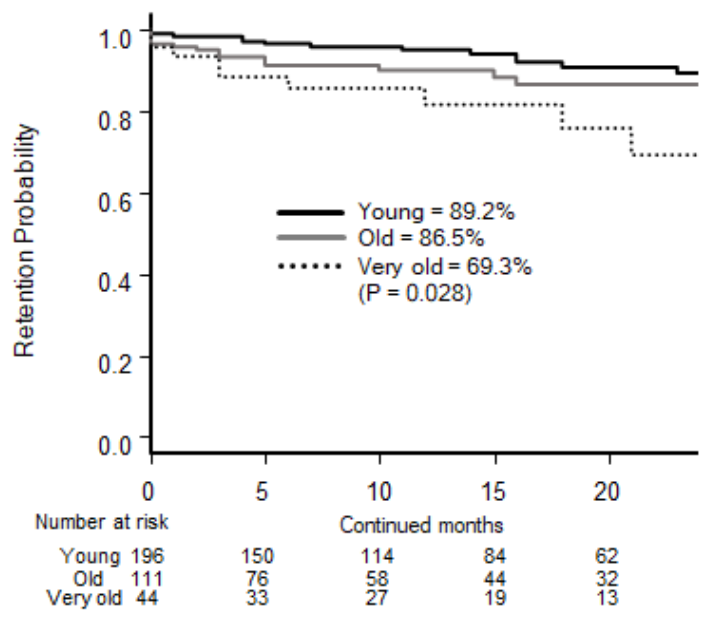

d

Drug retention due to toxic adverse events

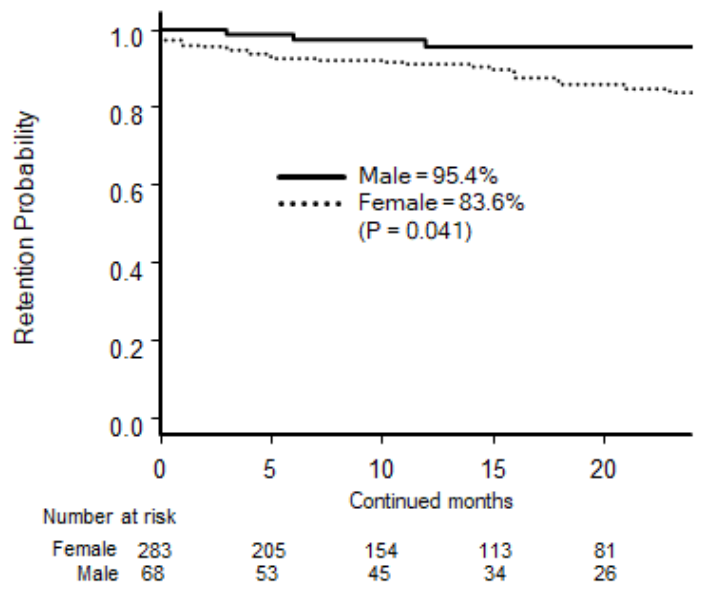

\section{Figure 1}

Adjusted drug retention by age and sex Adjusted drug retention between young ( $<65$ years), aged ( $65-74$ years), and very old ( $\geq 75$ years) groups, due to (a) lack of effectiveness and (b) toxic adverse events, and adjusted drug retention between male and female, due to (c) lack of effectiveness and (d) toxic adverse events. 


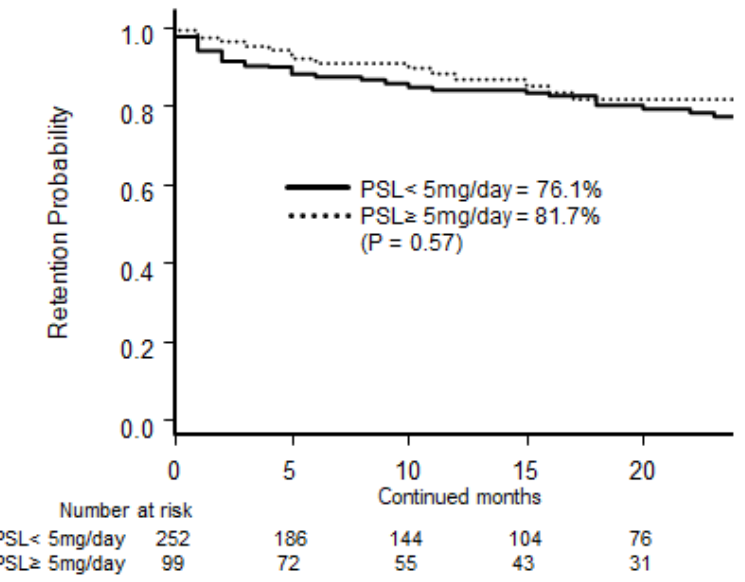

c Drug retention due to lack of effectiveness

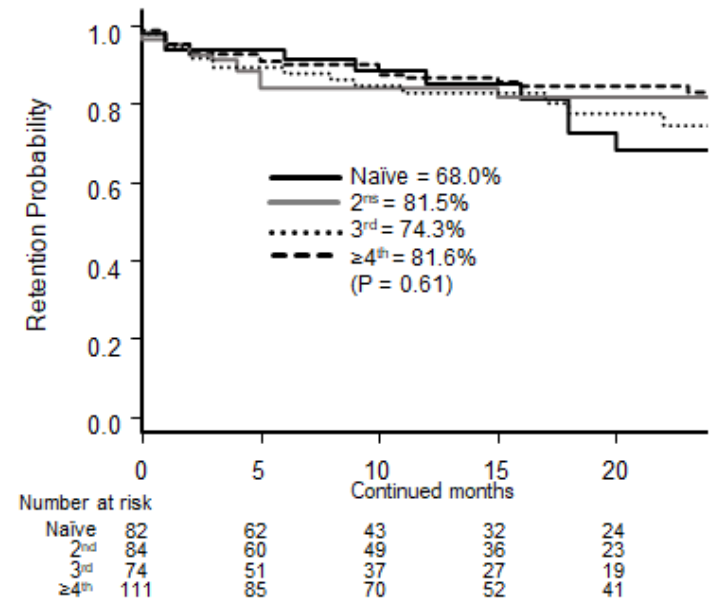

b

Drug retention due to toxic adverse events

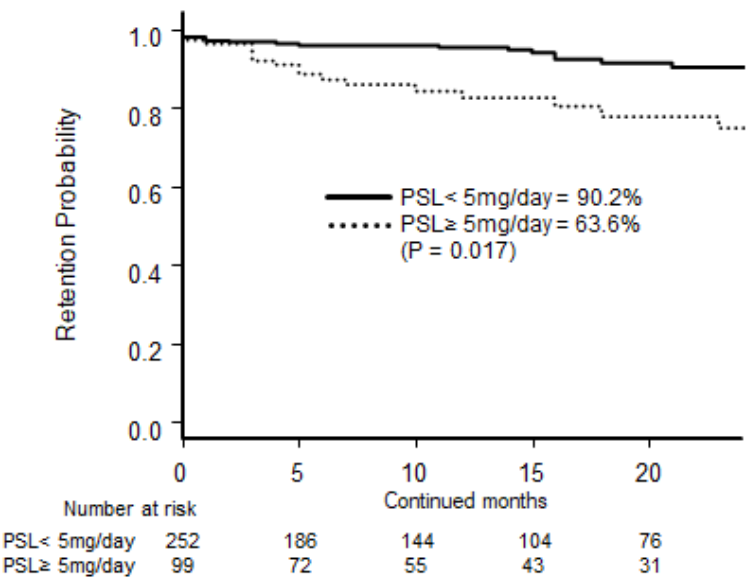

d

Drug retention due to toxic adverse events

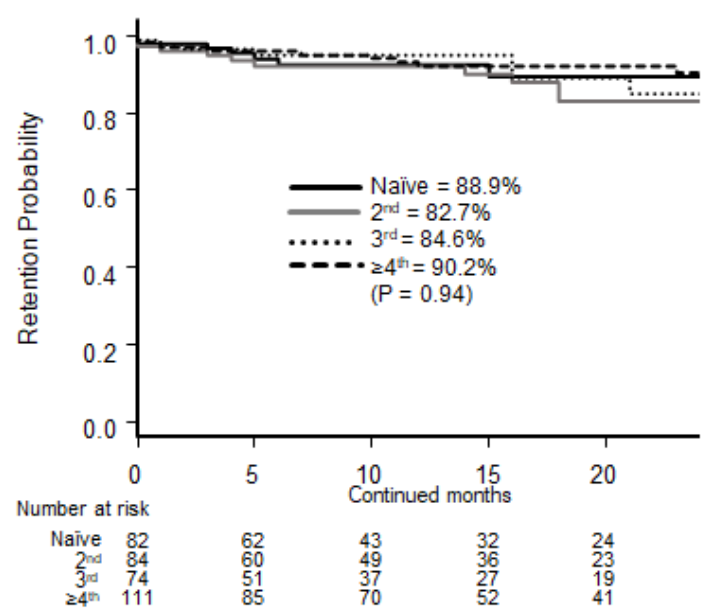

Figure 2

Adjusted drug retention by PSL dose and bDMARD/JAKi therapy Adjusted drug retention between concomitant PSL $<5 \mathrm{mg} /$ day and PSL $\geq 5 \mathrm{mg} /$ day, due to (a) lack of effectiveness and (b) toxic adverse events, and adjusted drug retention between switched bDMARD/JAKi groups due to (c) lack of effectiveness and (d) toxic adverse events. PSL, prednisolone; bDMARD, biological disease-modifying antirheumatic drug; JAKi, Janus kinase inhibitor. 
a

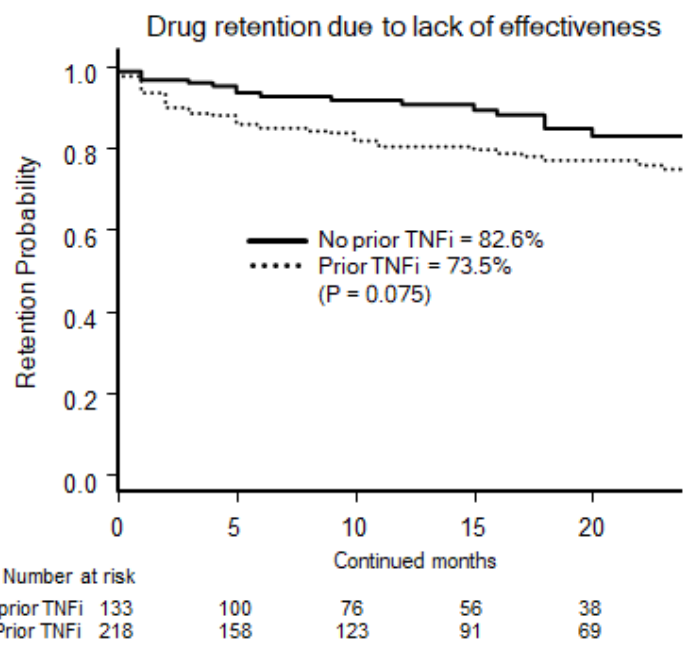

C

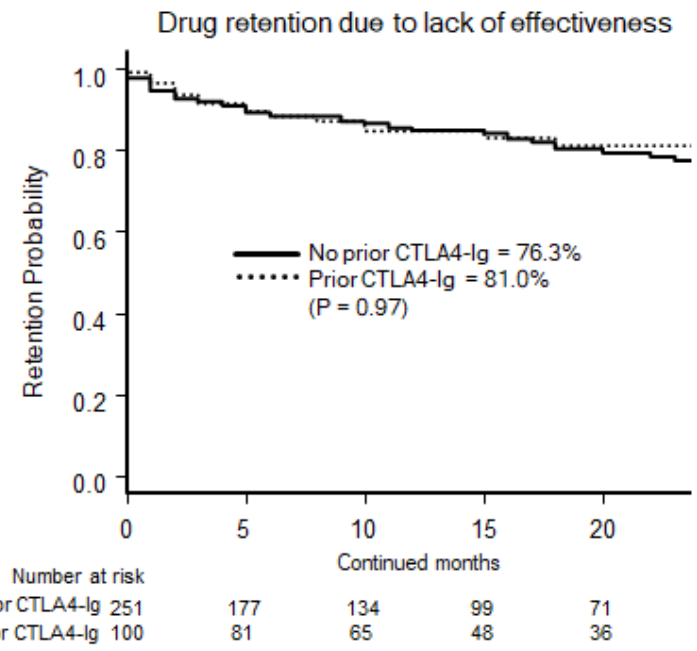

b

Drug retention due to lack of effectiveness

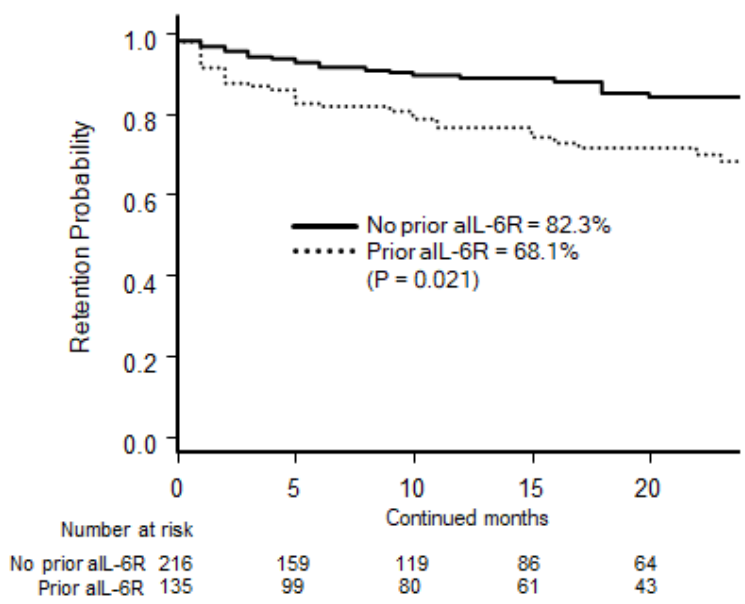

d Drug retention due to lack of effectiveness

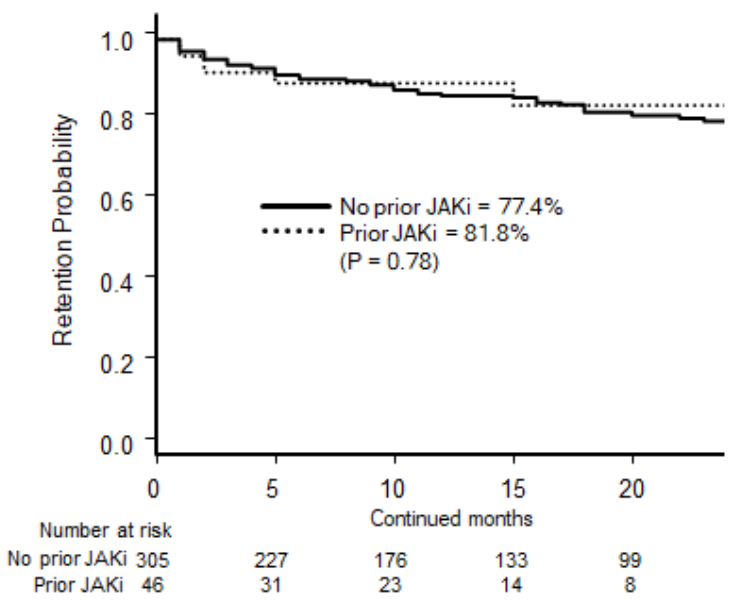

\section{Figure 3}

Adjusted drug retention by TNFi, alL-6R, CTLA4-Ig and JAKi experience Adjusted drug retention due to lack of effectiveness between (a) non-TNFi-experienced and TNFi-experienced groups, (b) non-alL-6Rexperienced and alL-6R-experienced groups, (c) non-CTLA4-Ig-experienced and CTLA4-Ig -experienced groups, (d) and non-JAKi-experienced and JAKi-experienced groups. TNFi, tumour necrosis factor inhibitor; alL-6R, anti-interleukin-6 receptor; CTLA4-Ig, cytotoxic T lymphocyte-associated antigen-4-Ig; JAKi, Janus kinase inhibitor. 